\title{
Exotic Higgs Scenarios
}

\author{
John F. Gunion*† \\ Department of Physics, U.C. Davis, Davis CA 95616 \\ E-mail: 'gunionephysics.ucdavis.edu'
}

ABStRACT: I briefly review the theoretical basis and experimental phenomenology of a number of exotic Higgs scenarios.

\section{Introduction}

There are so many models of electroweak symmetry breaking (EWSB) that it is impossible to review more than a few of them. My focus will be on theories in which there are "true" CP-even Higgs bosons emerging from the EWSB mechanism. I will first consider possible motivations for and problems associated with simply adding additional singlet, doublet or triplet Higgs representations to the Standard Model (SM) (i.e. without supersymmetry or extra dimensions). Next, I will discuss a few issues regarding the inclusion of $\mathrm{CP}$ violation and/or adding singlets to the MSSM. I will make a few remarks regarding Higgs bosons in the Left-Right Symmetric SUSY (SUSYLR) Model. I will then turn to models with extra dimensions, focusing on mixing between the Higgs boson and the radion in the simplest one doublet model with a warped 5th dimension. In all of these extensions, detection and study of the scalar bosons is complicated by the possibilities that the physical eigenstates: (a) mix strongly; (b) overlap (within relevant experimental resolutions); and/or (c) decay into one another. I will not have space to discuss higgsless models or little Higgs models.

\section{Extending the SM Higgs sector}

There are no particular theoretical problems (or benefits) associated with adding one or more singlet representations to the SM. However, each additional complex singlet gives rise to one extra CP-even Higgs boson and one extra CP-odd Higgs boson. Consequently, discovery would most likely be much more challenging as a result of the possibilities (a)-(c) listed above.

${ }^{*}$ Speaker.

${ }^{\dagger}$ Supported in part by the U.S. Department of Energy. 


\begin{tabular}{|c|c|c|c|c|c|c|c|}
\hline$N_{1 / 2,1}$ & $N_{1 / 2,3}$ & $N_{0,2}$ & $N_{0,4}$ & $N_{1,0}$ & $N_{1,2}$ & $\alpha_{s}$ & $M_{U}(\mathrm{GeV})$ \\
\hline 1 & 0 & 0 & 2 & 0 & 0 & 0.106 & $4 \times 10^{12}$ \\
1 & 0 & 4 & 0 & 0 & 1 & 0.112 & $7.7 \times 10^{12}$ \\
1 & 0 & 0 & 0 & 0 & 2 & 0.120 & $1.6 \times 10^{13}$ \\
2 & 0 & 0 & 0 & 1 & 0 & 0.116 & $1.7 \times 10^{14}$ \\
2 & 0 & 2 & 0 & 0 & 2 & 0.116 & $4.9 \times 10^{12}$ \\
2 & 1 & 0 & 0 & 0 & 2 & 0.112 & $1.7 \times 10^{12}$ \\
3 & 0 & 0 & 0 & 0 & 1 & 0.105 & $1.2 \times 10^{13}$ \\
\hline
\end{tabular}

Table 1: Representation choices that yield coupling constant unification are shown, where $N_{T, Y}$ is the number of representations of a given type. The required value of $\alpha_{s}$ (at one loop) and the unification scale $M_{U}$ are given for each case.

Adding additional doublets to the SM is also entirely possible. Doublets do not destroy the prediction of $\rho=1$, but Higgs sector potential parameters must be chosen so that $m_{H^{ \pm}}^{2}>0$. Each extra doublet adds one extra CP-even Higgs boson, one extra CP-odd Higgs boson and a charged Higgs pair. Discovery will generally be more difficult.

On can also consider adding triplet representations of various types to the SM. As discussed shortly, they could be good for coupling constant unification. If the vev of any neutral member of the triplet is zero, then this vev will remain zero at higher orders and the $\rho=1$ prediction will be retained. However, if the neutral member vev is non-zero $\rho$ is infinite at one loop and must be renormalized [i] that $\rho=1$ at tree-level. The value of $\rho$ becomes another input parameter to the theory. (Equivalently, the values of $m_{W}$ and $m_{Z}$ are independently renormalized parameters.)

One should recall that $Y \neq 0$ triplets are motivated by left-right (LR) symmetric models within which context they can generate neutrino masses via the seesaw mechanism [2i] Aside from the triplet, an L-R model must contain at least one doublet and more are certainly a possibility.

As already noted, unification of the coupling constants can be achieved without SUSY by adding additional Higgs representations to the SM (see, for example, Ref. [3] 30 ). Some simple choices are illustrated in Table 1in In all cases, the resulting value of $M_{U}$ is smaller than is comfortable for proton decay. However, this is not necessarily a problem. For example, coupling unification need not imply true group unification, as, for example, in certain types of string models. My favorite model is $N_{\frac{1}{2}, 1}=2, N_{1,0}=1$, leading to $\alpha_{s}\left(m_{Z}\right)=0.115$ and $M_{U}=1.7 \times 10^{14} \mathrm{GeV}$. Let me denote the triplet members by $\xi^{+, 0,-}$ and define $v_{T} \equiv\left\langle\xi^{0}\right\rangle$. For the doublet members, we define $v_{u} \equiv\left\langle H_{u}\right\rangle, v_{d} \equiv\left\langle H_{d}\right\rangle$ and $v_{D}=\sqrt{v_{u}^{2}+v_{d}^{2}}$. Further, we use the notation $\tan \beta=v_{u} / v_{d}$ and $\tan \gamma=v_{T} / v_{D}$.

A very interesting observation is that for this (and other) models, precision electroweak (PEW) constraints do not imply that there must be a discoverable light Higgs boson. For example, consider the case of $v_{T}=0$ [i] they will give rise to coupling constant unification but they will not be detectable at the LHC or LC. Further, for $\tan \beta$ moderate in size and $200 \mathrm{GeV} \lesssim m_{A^{0}}$, one is in the LHC

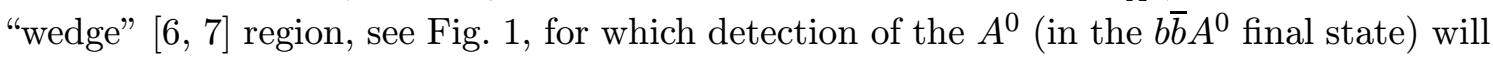


not be possible at the LHC. At the LC, the moderate $\tan \beta$ wedge for which $A^{0}$ detection

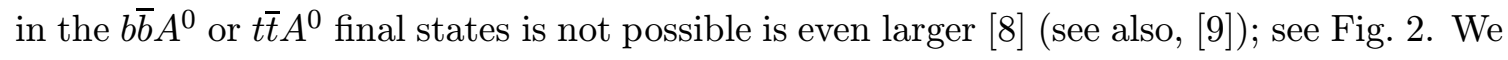
also note that $A^{0} A^{0} \nu \bar{\nu}$ production at most allows detection of the $A^{0}$ up to $m_{A^{0}} \sim 285 \mathrm{GeV}$ for $\sqrt{s}=800 \mathrm{GeV}$ LC operation [i]



Figure 1: $5 \sigma$ discovery contours for MSSM Higgs boson detection in various channels are shown in the $\left[m_{A^{0}}, \tan \beta\right]$ parameter plane, assuming maximal mixing and an integrated luminosity of $L=300 \mathrm{fb}^{-1}$ for the ATLAS detector. The "wedge" region for the $A^{0}$ alone, i.e. without the degenerate $H^{0}$, would be somewhat larger.

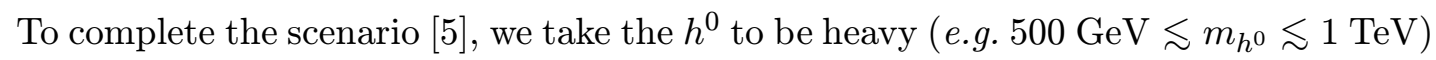
and SM-like and choose $m_{H^{0}}$ and $m_{H^{ \pm}}$still larger (but $\lesssim 1.2 \mathrm{TeV}$ in order that that the Higgs potential quartic couplings are perturbative) with $m_{H^{ \pm}}-m_{H^{0}}>0$ but quite small (e.g. $\sim$ few GeV). A heavy SM-like $h^{0}$ leads to large $\Delta S>0$ and $\Delta T<0$ contributions, but these are compensated by a large $\Delta T>0$ from $m_{H^{ \pm}}-m_{H^{0}}>0$ :

$$
\Delta \rho=\frac{\alpha}{16 \pi m_{W}^{2} c_{W}^{2}}\left\{\frac{c_{W}^{2}}{s_{W}^{2}} \frac{m_{H^{ \pm}}^{2}-m_{H^{0}}^{2}}{2}-3 m_{W}^{2}\left[\log \frac{m_{h^{0}}^{2}}{m_{W}^{2}}+\frac{1}{6}+\frac{1}{s_{W}^{2}} \log \frac{m_{W}^{2}}{m_{Z}^{2}}\right]\right\} .
$$

The result is to predict $S, T$ values that are well within the usual 90\% CL PEW region, as

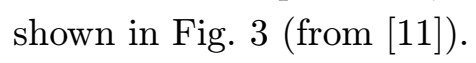

In this model, the LHC would discover a SM-like $h^{0}$ with $m_{h^{0}}$ well above the roughly $200 \mathrm{GeV}$ upper limit obtained in the strictly SM context. Such an $h^{0}$ would be beyond the reach of an LC with $\sqrt{s} \lesssim m_{h^{0}}+2 m_{Z}$. Without much higher LC $\sqrt{s}$, there are only two options for clarifying the situation. First, Giga- $Z$ PEW measurements (combined with $\Delta m_{W}=6 \mathrm{MeV}$ from a $W W$ threshold scan) would pinpoint the $S, T$ values. Sample Giga- 




Figure 2: The stars outline the wedge within which $A^{0}$ detection in $e^{+} e^{-} \rightarrow t \bar{t} A^{0}$ or $b \bar{b} A^{0}$ will not be possible. The +'s show points with $>4 \sigma$ signal in $\gamma \gamma \rightarrow A^{0} \rightarrow b \bar{b}$ after two years of carefully configured NLC operation in the $\gamma \gamma$ collider mode at $\sqrt{s}_{e e}=630 \mathrm{GeV}$. The $o$ 's show additional points that would give a $>4 \sigma$ signal after two years of TESLA operation in the $\gamma \gamma$ collider mode. (From [1] $\left.\overline{1}_{2}\right] \cdot$ )



Figure 3: Outer ellipses: 90\% CL region for $U=0$ and $m_{h_{\mathrm{SM}}}=115 \mathrm{GeV}$. Blobs: $S, T$ predictions for the model described in text with $m_{h^{0}}=500 \mathrm{GeV}$ (left) or $m_{h^{0}}=800 \mathrm{GeV}$ (right) and $m_{H^{ \pm}}-m_{H^{0}}$ chosen to minimize the PEW $\Delta \chi^{2}$. Innermost (middle) ellipse: $90 \%$ (99.9\%) CL region for $m_{h_{\mathrm{SM}}}=$ $115 \mathrm{GeV}$ after Giga- $Z$ PEW measurements and a $\Delta m_{W} \lesssim 6 \mathrm{MeV}$ threshold scan measurement. Stars: SM $S, T$ prediction if $m_{h_{\mathrm{SM}}}=500$ or $800 \mathrm{GeV}$.

$Z$ error ellipses for $S, T$ are shown in Fig. ${ }_{-1}^{3}$. Second, $\gamma \gamma$ collisions could allow $A^{0}$ discovery in the wedge [i] $\overline{2} \overline{2}] ;$; see Fig. $\overline{2}$.

Finally, it is amusing to note that for $v_{T}=0$, the lightest of the triplet Higgs members would be stable. It is easily arranged that the lightest is the $\xi^{0}$, thereby providing a viable 
dark matter candidate.

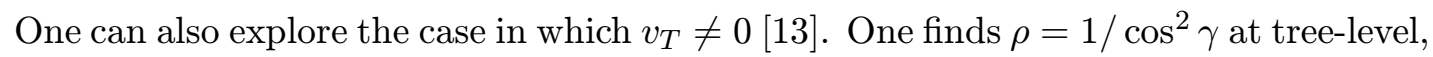
which for small $\gamma$ means $\alpha \Delta T \sim+\gamma^{2}$. Meanwhile, $\Delta S=0$ for a $Y=0$ triplet. In other words, this is another source of isospin breaking that (at tree-level) allows a heavier $m_{h^{0}}$. If $m_{h^{0}} \sim 1 \mathrm{TeV}$ and $m_{H^{ \pm}}=m_{H^{0}}$, then $\gamma \sim 0.06$ gives acceptable PEW. However, I again emphasize that this tree-level result is infinitely renormalized at one-loop and there is no actual prediction for $\rho$; it becomes another input to the renormalization procedure for the model for which a value must be specified in terms of, for example, the LEP measurement.

While extending the SM simply by adding to the Higgs sector allows for coupling constant unification and for the possibility of heavier and more difficult-to-detect Higgs bosons, such extensions do not provide a solution to the hierarchy and fine-tuning problems. These difficulties can at most be delayed. For example, if the Higgs mass is adjusted relative to the top, $W, Z, \ldots$ so as to satisfy the Veltman conditions (or perhaps their generalization to higher orders) the hierarchy problem can be postponed until scales of order $10 \mathrm{TeV}$ [i] $\left.\mathbf{1}^{1} \mathrm{i}\right]$. Little Higgs models [i] $\left[\overline{1} \overline{1}\right.$ ] delay the problems by the introduction of $W^{\prime}, Z^{\prime}, t^{\prime}, \ldots$, with couplings carefully adjusted to cancel the SM quadratic divergences at one loop. The phenomenology of these scalars (see, for example, Ref. [1] 16 i $]$ ) will not be pursued here.

The only way to solve the hierarchy and fine-tuning problems all the way up to the Planck scale, while having a true Higgs sector with some CP-even Higgs bosons, is to consider models that incorporate supersymmetry and/or large-scale extra dimensions.

\section{Extensions of the MSSM Higgs sector}

The attractive features of the minimal supersymmetric model (MSSM), containing exactly two Higgs doublets, are well known (see [1] $\left.\bar{z}_{1}\right]$ and references therein). In particular, the MSSM yields nearly exact coupling constant unification and automatic EWSB via radiative evolution. However, the CP-conserving (CPC) MSSM is being pushed into an uncomfortable corner in several ways. First, the rather substantial lower bound on the mass of the light $h^{0}$ from LEP [1] $\left.\overline{8}_{1}\right]$ is only easily accommodated in the restrictive part of the MSSM parameter space characterized by large $\tan \beta$ combined with large top squark masses and mixing. There are also significant direct lower bounds on the mass of the lightest stop. The current conclusion is that this part of parameter space cannot be reconciled with that for which the CP-conserving (CPC) MSSM provides adequate baryogenesis. A brief review

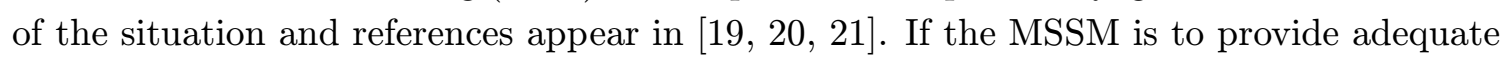
dark matter as well as be consistent with $b \rightarrow s \gamma$ and $g_{\mu}-2$, further constraints are placed on the MSSM parameter space. An early discussion of the tension between the dark matter and baryogenesis requirements appears in [202i]. Most recently, the most probable portions of parameter space consistent with Higgs mass limits, dark matter, $b \rightarrow s \gamma$ and $g_{\mu}-2$ have been delineated within the constrained MSSM (cMSSM) in [ī $\overline{2} \overline{3}$. One finds an appropriate amount of dark matter in the coannihilation, rapid-annihilation and focus regions, which are for the most part at high $m_{0}$. However, a more general study is needed to ascertain the implications of dark matter for baryogenesis since the cMSSM boundary conditions do not allow for a very light right-handed stop and very heavy left-handed stop, as required 
to have significant baryogenesis. (Of course, any conflict could be evaded if the slepton masses entering into dark matter calculations and the stop masses entering into baryogenesis were treated differently.) A final problem for the MSSM is that no really attractive source for the superpotential $\mu$ parameter has been proposed. Most explanations involve some extension of the MSSM.

Keeping to the supersymmetric context, but going beyond the MSSM, the above issues have led to consideration of: (1) introducing CP-violation (CPV) into the MSSM Higgs sector (from CP-violating soft-SUSY loops) [24] ; (2) the next-to-minimal supersymmetric model (NMSSM) in which one extra singlet superfield is added to the MSSM [205in] thereby allowing a natural explanation for the $\mu$ parameter (see [i $\left[\begin{array}{l}1 \\ 7_{1}\end{array}\right]$ for a discussion and early references); (3) taking seriously the prediction common to many string models of many


tensions, such as the recent ideas of [i2 $\overline{2} \overline{7}]$, will not be discussed here. A common feature of all of these extensions is that they lead to possible difficulties for detecting even one of the supersymmetric Higgs bosons at the LHC. In particular, one can choose parameters so that the following problems arise (which we summarized very briefly earlier in the non-supersymmetric context).

- The easily produced Higgs boson(s), e.g. those with large $W W / Z Z$ coupling, can

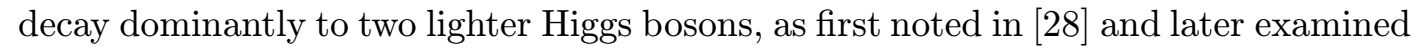

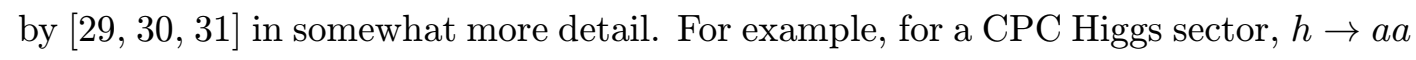
and $h^{\prime} \rightarrow h h$ decays are both possible in general. In the case of a CPV Higgs sector, general decays of the type $h \rightarrow h^{\prime} h^{\prime \prime}$ are allowed. In addition, $h \rightarrow h^{\prime} V$ decays are generically present, although they tend to be much less dangerous than the Higgs to Higgs-pair decays.

In both the CPC and CPV cases, the Higgs potential can be such that these lighter Higgs bosons have $W W / Z Z$ couplings that are very weak or zero (e.g. they can be pseudoscalars in the CPC case) while at the same time their Yukawa couplings to $t \bar{t}$ and $b \bar{b}$ are not very different from SM-like values. In this case, it will typically be very difficult to detect them directly.

- When there are multiple mixed CP-even Higgs bosons in a CPC Higgs sector or mixed CP-even and CP-odd Higgs bosons in a CPV Higgs sector, the Higgs bosons will generically tend to share the $W W / Z Z$ coupling strength. At the LHC, this leads to a corresponding reduction of the $W$-loop contribution to the $h \gamma \gamma$ couplings which will then strongly cancel against the $t$-loop contribution resulting in a dramatic decrease in the rate for the excellent resolution $g g \rightarrow h \rightarrow \gamma \gamma$ channels. In addition, the $g g \rightarrow h \rightarrow Z Z^{*} \rightarrow 4 \ell$ rate is also suppressed relative to the poorer resolution $b \bar{b}$ and $t \bar{t}$ channel branching ratios (not to mention any possible $h \rightarrow V h^{\prime}$ or $h \rightarrow h^{\prime} h^{\prime \prime}$ decays).

- In addition, the Higgs bosons can differ in mass so that signals in, for example, $g g \rightarrow t \bar{t} h$ with $h \rightarrow b \bar{b}$ or $h \rightarrow \tau^{+} \tau^{-}$are overlapping as well as reduced in magnitude. Such overlaps obviate many of the standard discovery modes. 
Even in the absence of $h$ decays to other Higgs bosons, the $W W \rightarrow h \rightarrow \tau^{+} \tau^{-}$ detection channel will take a "double-hit". First, the production rate for each $h$ is suppressed due to reduced $W W h$ coupling. Second, the poor mass resolution in the $\tau^{+} \tau^{-}$channel implies that the signals for different $h$ 's (separated in mass by, say, $10 \mathrm{GeV}$ ) will overlap and make peak detection impossible. Instead, one must try to determine the presence of a broad excess in the $M_{\tau \tau}$ distribution.

If these problems result in the LHC failing to detect a signal for any of the Higgs bosons, the LC can still succeed in searching for the $h$ using $e^{+} e^{-} \rightarrow Z h$ production by looking for a bump, or at least a broad enhancement, in the reconstructed $M_{X}$ mass distribution in the inclusive $e^{+} e^{-} \rightarrow Z X$ channel. Even if the signals from different Higgs bosons overlap somewhat and their strength is maximally shared, the excess in the $M_{X}$ distribution will be apparent at the LC so long as a reasonable fraction of the overlapping Higgs bosons with good $W W / Z Z$ coupling are not too heavy relative to the $\mathrm{LC} \sqrt{s}$. And, of course, the inclusive $M_{X}$ peak or broad excess is independent of how the Higgs bosons decay.

Even in this very difficult situation, the LHC will have played an important role. If light Higgs bosons more or less saturate the $W W / Z Z$ coupling $\left(\sum_{i} g_{h_{i} W W}^{2}=g_{h_{S M} W W}^{2}\right)$, $W_{L} W_{L} \rightarrow W_{L} W_{L}$ scattering will be perturbative at the LHC. Observation of this perturbativity at the LHC will imply that such light Higgs (or some other type of perturbative EWSB) are present below the $\mathrm{TeV}$ scale, implying the absolute need for a linear collider to observe them.

\subsection{CP-violating MSSM}

A first case in point is the CP-violating MSSM. If the soft-supersymmetry-breaking parameters (such as the gluino mass, the $\mu$ parameter, and the $A$ parameters) are allowed to be complex, significant CP violation is induced in the Higgs potential at the one-loop level. Portions of the CPV MSSM parameter space with large phases for the $\mu$ parameter

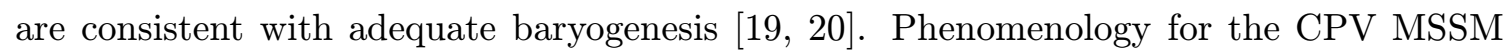
is studied in $[\overline{3} \overline{2}]$. A survey of the allowed possibilities reveals a particularly nasty case, dubbed the CPX scenario, in which there are points in the $\left(m_{H^{ \pm}}, \tan \beta\right)$ parameter space for which there are light Higgs bosons that would not have been discovered at LEP and that will not be detected at the Tevatron or LHC. A linear collider is required for their detection. They escape LHC and LEP detection by virtue of a combination of the reduced $W W / Z Z$ coupling, Higgs to Higgs decays, and overlapping of resonances mentioned earlier. While the CPX scenario allows for adequate baryogenesis (by virtue of more sources of $\mathrm{CP}$ violation and lighter Higgs bosons as compared to the MSSM), the $\mu$ parameter remains a problematic issue.

\subsection{The next-to-minimal supersymmetric model}

In the NMSSM, the MSSM superpotential term $\mu \widehat{H}_{1} \widehat{H}_{2}$ in the MSSM is replaced by

$$
\lambda \widehat{H}_{1} \widehat{H}_{2} \widehat{S}+\frac{\kappa}{3} \widehat{S}^{3} .
$$


The superpotential is thus scale invariant and $\mu_{\mathrm{eff}}=\lambda\langle S\rangle$. It seems apparent that the increased freedom in the Higgs and other sectors will allow for adequate baryogenesis and appropriate dark matter without the necessity to introduce $\mathrm{CP}$ violation, either directly into the Higgs sector or indirectly at the loop-level through CP-violating soft parameter phases. However, no explicit study has been performed.

That the Higgs bosons of the CPC NMSSM might evade LEP constraints and remain undiscovered at the Tevatron and LHC was first pointed out at Snowmass96 [2] $2 \overline{8}]$. By the time of Snowmass01, improvements had been made in the standard LHC discovery modes and several additional modes had been developed in detail. In [30 $\left.{ }^{0}\right]$ the issue of Higgs discovery in the NMSSM was reexamined. I give a few details regarding that study. First, the parameter space was explored without making any assumption regarding the soft-SUSY-breaking terms. In particular, all five soft supersymmetry breaking terms in the potential

$$
m_{H_{1}}^{2} H_{1}^{2}+m_{H_{2}}^{2} H_{2}^{2}+m_{S}^{2} S^{2}+\lambda A_{\lambda} H_{1} H_{2} S+\frac{\kappa}{3} A_{\kappa} S^{3}
$$

were considered as independent. In the stop sector, we chose the soft masses $m_{Q}=m_{T} \equiv$ $M_{\text {susy }}=1 \mathrm{TeV}$ and scanned over $X_{t} \equiv 2 \frac{A_{t}^{2}}{M_{\text {susy }}^{2}+m_{t}^{2}}\left(1-\frac{A_{t}^{2}}{12\left(M_{\text {susy }}^{2}+m_{t}^{2}\right)}\right)$. As in the MSSM, the value $X_{t}=\sqrt{6}$ - so called maximal mixing - maximizes the radiative correction to the light Higgs boson masses. It leads to the most challenging points in NMSSM parameter space. We required consistency with LEP constraints on Higgs bosons and supersymmetric particles. We also required $m_{h^{ \pm}}>155 \mathrm{GeV}$, so that $t \rightarrow h^{ \pm} b$ would not be seen. Finally, we examined only parameter space points for which there were no Higgs to SUSY or Higgs to Higgs decays.

We examined the "usual" LHC discovery modes using the latest experimental simulation results by the ATLAS and CMS collaborations. The modes are:

1) $g g \rightarrow h / a \rightarrow \gamma \gamma$

2) associated $W h / a$ or $t \bar{t} h / a$ production with $\gamma \gamma \ell^{ \pm}$in the final state;

3) associated $t \bar{t} h / a$ production with $h / a \rightarrow b \bar{b}$;

4) associated $b \bar{b} h / a$ production with $h / a \rightarrow \tau^{+} \tau^{-}$;

5) $g g \rightarrow h \rightarrow Z Z^{(*)} \rightarrow 4$ leptons;

6) $g g \rightarrow h \rightarrow W W^{(*)} \rightarrow \ell^{+} \ell^{-} \nu \bar{\nu}$

7) $W W \rightarrow h \rightarrow \tau^{+} \tau^{-}$

8) $W W \rightarrow h \rightarrow W W^{(*)}$.

For each mode and each NMSSM Higgs boson, we estimated the expected statistical significance at the LHC by rescaling results for the SM Higgs boson and/or the the MSSM $h, H$ and/or $A$. Note that the $t \bar{t} h \rightarrow t \bar{t} b \bar{b}$ mode was quite important. We had the experimentalists extrapolate this beyond the usual SM mass range of interest. For each mode, our procedure was to use the results for the "best detector" (e.g. CMS for the $t \bar{t} h$ channel), assuming $L=300 \mathrm{fb}^{-1}$ for that one detector. The result is simply stated: at least one of the NMSSM Higgs bosons will be detected at the LHC.

However, we also found [3i in that this optimistic conclusion was no longer valid once points in the model parameter space were included for which Higgs to Higgs decays are 


\begin{tabular}{|c|c|c|c|c|c|c|}
\hline Point \# & 1 & 2 & 3 & 4 & 5 & 6 \\
\hline \hline$m_{h}(\mathrm{GeV})$ & 115 & 119 & 123 & 118 & 134 & 130 \\
\hline$m_{a_{1}}(\mathrm{GeV})$ & 56 & 7 & 35 & 41 & 59 & 7 \\
\hline LHC $S / \sqrt{B}$ for $L=300 \mathrm{fb}^{-1}$ & 50 & 22 & 69 & 63 & 62 & 21 \\
\hline
\end{tabular}

Table 2: We give values for $m_{a_{1}}$ and $m_{h}$, where the SM-like Higgs is $h=h_{1}$ for points 1,2 and 3 and $h=h_{2}$ for points 4,5 , and 6 . The final LHC $S / \sqrt{B}$ results (after all cuts) for each point are given assuming integrated luminosity of $L=300 \mathrm{fb}^{-1}$ and integration over the interval $40 \mathrm{GeV} \leq M_{j j \tau^{+} \tau^{-}} \leq 150 \mathrm{GeV}$.

allowed (but still excluding points for which Higgs decays to superparticles are significant). In particular, we found cases for which all the modes 1) - 8) give very weak signals for all Higgs bosons. All such points have certain common properties. First, either the $h_{1}$ or $h_{2}$ is SM-like and has a mass between 115 and $135 \mathrm{GeV}$ (i.e. above the LEP limit, but still quite light). We denote it by $h$, with the other of the two lighter CP-even Higgs bosons, $h_{2}$ or $h_{1}$, respectively, denoted by $h^{\prime}$. (The $h_{3}$ and $a_{2}$ are always very heavy and not detectable at the LHC nor at a linear collider with $\sqrt{s} \lesssim 800 \mathrm{GeV}$, and will not be discussed further.) Second, the $h$ state decays dominantly to a pair of light CP-odd states, $a_{1} a_{1}$, with $m_{a_{1}}$ between 5 and $65 \mathrm{GeV}$. Third, $\tan \beta$ is moderate in size so that the $t \bar{t}+\left(h, h^{\prime}, a_{1}\right)$ rates are somewhat suppressed while the $b \bar{b}+\left(h, h^{\prime}, a_{1}\right)$ production rates are insufficiently enhanced to allow Higgs detection. Finally, since the $h^{\prime}$ and $a_{1}$ have small or no $W W / Z Z$ coupling, detection modes related to this coupling are not viable, including the $g g \rightarrow h^{\prime}, a_{1} \rightarrow \gamma \gamma$ modes. Detecting any of the Higgs bosons at the LHC would seem to necessitate the development of techniques for extracting a signal for the $W W \rightarrow h \rightarrow a_{1} a_{1}$ mode. We have focused on the $W W \rightarrow h \rightarrow a_{1} a_{1} \rightarrow j j \tau^{+} \tau^{-}$final state (where $j j=b \bar{b}$ for $m_{a_{1}}>10 \mathrm{GeV}$ ), with $\tau^{+} \tau^{-} \rightarrow \ell^{+} \ell^{-} \nu \bar{\nu}$. The invariant mass $M_{\tau^{+} \tau^{-}}$is reconstructed by resolving the missing momentum from the neutrinos along the $\ell^{+}, \ell^{-}$directions. After many cuts, including forward / backward jet tagging, requiring approximately equal mass for the $\tau^{+} \tau^{-}$pair and the $j j$ pair, and various vetoes, but without $b$-tagging, we get the signals shown in Fig. ${ }_{14}^{1}$ relative to the backgrounds for 6 typical cases of the type described. The masses $m_{h}$ and $m_{a_{1}}$ for each of the 6 cases are tabulated in Table $\underline{\underline{2}}$.

For all six NMSSM setups, the Higgs resonance produces a bump at low $M_{j j \tau^{+} \tau^{-}}$, well above the dominant $t \bar{t}$ background. The potentially difficult $\tau^{+} \tau^{-}+$jets DY background has been more or less eliminated by the strong cuts requiring 2 fast forward / backward jets +2 softer jets.

Since the main surviving background is from $t \bar{t}$ production, $b$ tagging is not helpful. For points 2 and 6 , for which the signal has no $b$ 's in the final state, anti-b-tagging might be useful, but has not been considered here.

To estimate $S / \sqrt{B}$, we assume $L=300 \mathrm{fb}^{-1}$, a $K$ factor of 1.1 for the $W W$ fusion signal and $K$ factors of 1,1 and 1.6 for the DY $\tau^{+} \tau^{-}, Z Z$ production and $t \bar{t}$ backgrounds,

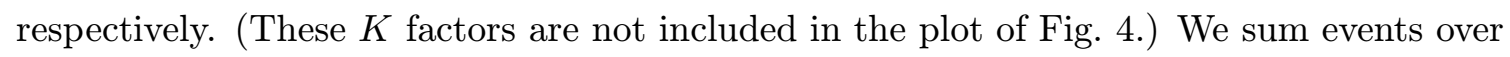
the region $40 \leq M_{j j \tau^{+} \tau^{-}} \leq 150 \mathrm{GeV}$. (Had we only included masses below $130 \mathrm{GeV}$, we would have had no $t \bar{t}$ background, and the $S / \sqrt{B}$ values would be enormous. However, we 


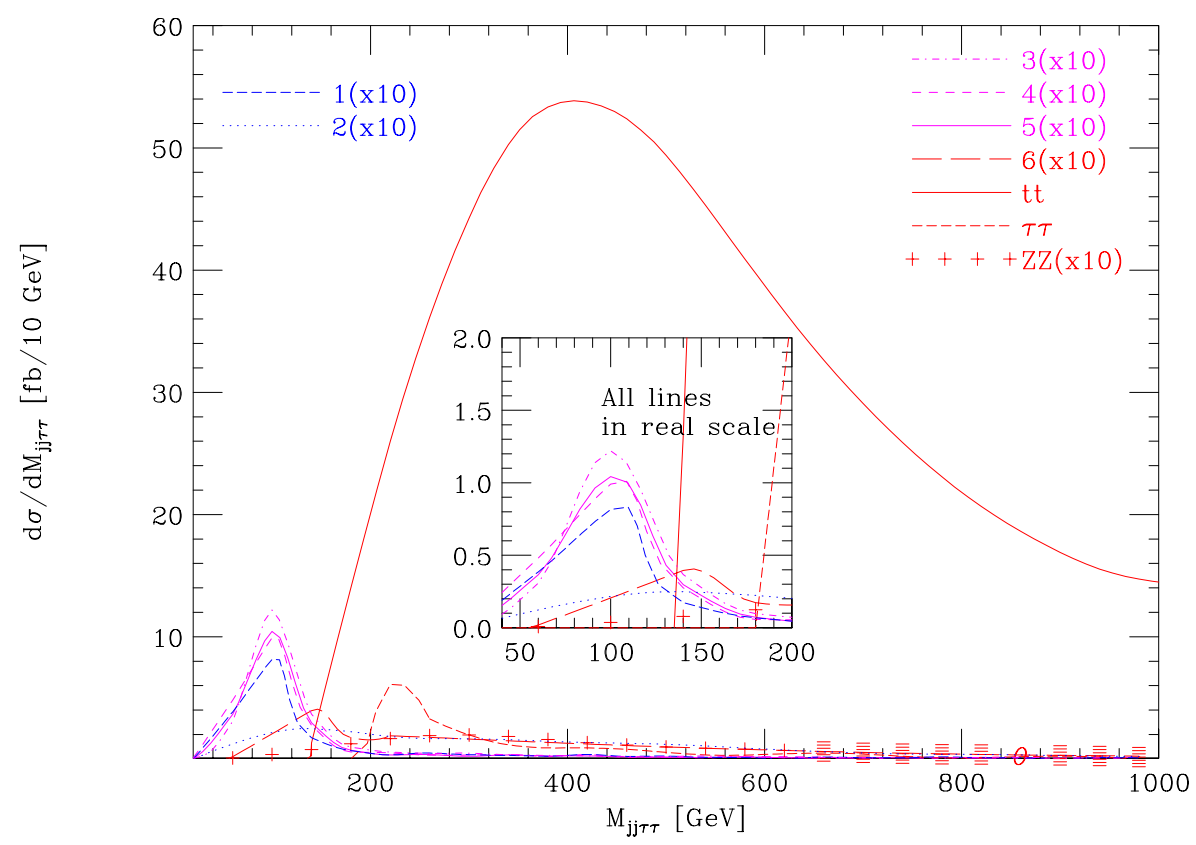

Figure 4: Reconstructed mass of the $j j \tau^{+} \tau^{-}$system for signals and backgrounds before $b$-tagging, at the LHC. We plot $d \sigma / d M_{j j \tau^{+} \tau^{-}}[\mathrm{fb} / 10 \mathrm{GeV}]$ vs $M_{j j \tau^{+} \tau^{-}}[\mathrm{GeV}]$. The lines corresponding to points 4 and 5 are visually indistinguishable. No $K$ factors are included.

are concerned that this absence of $t \bar{t}$ background below $130 \mathrm{GeV}$ might be a reflection of limited Monte Carlo statistics. As a result we have taken the more conservative approach of at least including the first few bins for which our Monte Carlo does predict some $t \bar{t}$ background.)

For points 1, 2, 3, 4, 5 and 6 , we obtain signal rates of about $S=1636,702,2235,2041$, 2013 , and 683 , respectively. The $t \bar{t}+$ jets background rate is $B_{t t} \sim 795$. The $Z Z$ background rate is $B Z Z \sim 6$. The DY $\tau^{+} \tau^{-}$background rate is negligible. (We are continuing to increase our statistics to get a fully reliable estimate.) The resulting $N_{S D}=S / \sqrt{B}$ values for points 1-6 are 50,22,69,63,62, and 21 , respectively. The smaller values for points 2 and 6 are simply a reflection of the difficulty of isolating and reconstructing the two jets coming from the decay of a very light $a_{1}$. Overall, these preliminary results are very encouraging and suggest that a no-lose theorem for NMSSM Higgs detection at the LHC is close at hand.

What about the LC? Since the low- $M_{j j \tau^{+} \tau^{-}}$bump would be the only Higgs boson signal at the LHC, the LC will be essential for confirming and clarifying the nature of the LHC signal. Because the $Z Z h$ coupling is nearly full strength in all cases, and because the $h$ mass is $\lesssim 140 \mathrm{GeV}$, discovery of the $h$ will be very straightforward via $e^{+} e^{-} \rightarrow Z h$ using the $e^{+} e^{-} \rightarrow Z X$ reconstructed $M_{X}$ technique which is independent of the "unexpected" complexity of the $h$ decay to $a_{1} a_{1}$. This will immediately provide a direct measurement of the $Z Z h$ coupling with very small error. The next stage will be to look at rates for the various $h$ decay final states, $F$, and extract $B R(h \rightarrow F)=\sigma\left(e^{+} e^{-} \rightarrow Z h \rightarrow Z F\right) / \sigma\left(e^{+} e^{-} \rightarrow Z h\right)$. For the NMSSM points considered here, the main channels would be $F=b \bar{b} b \bar{b}, F=b \bar{b} \tau^{+} \tau^{-}$ 
and $F=\tau^{+} \tau^{-} \tau^{+} \tau^{-}$(or analogues with $b \bar{b}$ replaced by $j j$ if $m_{a_{1}}<10 \mathrm{GeV}$ as for points 2 and 6$)$. At the LC, a fairly accurate determination of $B R(h \rightarrow F)$ should be possible in all three cases. This would allow us to check that $B R\left(a_{1} \rightarrow b \bar{b}\right) / B R\left(a_{1} \rightarrow \tau^{+} \tau^{-}\right)$is as expected based on couplings being proportional to mass, as required for $a_{1}$ to be a Higgs boson. We could then also determine $B R\left(h \rightarrow a_{1} a_{1}\right)$. We have also shown that $e^{+} e^{-} \rightarrow \nu \bar{\nu} W W$ followed by $W W \rightarrow h \rightarrow a a \rightarrow j j \tau^{+} \tau^{-}$always gives a good signal at the LC after cuts that essentially eliminate all backgrounds.

Overall, we are very close to establishing a no-lose theorem for NMSSM Higgs detection at the LHC. What remains is to include points for which $h \rightarrow \widetilde{\chi}_{1}^{0} \widetilde{\chi}_{1}^{0}$ and / or $a_{1} \rightarrow \widetilde{\chi}_{1}^{0} \widetilde{\chi}_{1}^{0}$ (invisible decays) are allowed. Clearly, if SUSY is discovered at the LHC and no Higgs bosons are detected in the standard MSSM Higgs search modes, a careful search for the signal we have considered should have a high priority. Eventually, we will need to consider a CP-violating NMSSM Higgs sector with five mixed Higgs!

\subsection{Multiple singlet superfields: a worst-case scenario}

Allowing for still more singlet (or doublet) superfields further complicates the possibilities for Higgs detection. In [ī $3 \overline{3}]$, a worst case scenario was developed. The basic idea is to allow for a very large number of Higgs fields spread out in a kind of continuum. Models that produce many extra neutral Higgs fields include fermionic constructions with 3 families [2]-i] by intervals of $\sim 10 \mathrm{GeV}$, i.e. of order the experimental mass resolution in the $b \bar{b}, \tau^{+} \tau^{-}$, $W W, a a$ and $X$ (meaning the recoil mass $M_{X}$ in the $e^{+} e^{-} \rightarrow Z X$ channel) final states. In the worst case, all the physical eigenstates should share roughly equally the $V V$ coupling strength.

To assess prospects for detecting such a continuum Higgs scenario, theoretical and PEW constraints are critical. We use continuum notation. The most important issue is the value of $m_{C}$ in

$$
\int_{0}^{\infty} d m K(m) m^{2}=m_{C}^{2}, \quad \text { where } \quad \int_{0}^{\infty} K(m)=1
$$

where $K(m)\left(g m_{W}\right)^{2}$ is the (density in Higgs mass of the) strength of the $h W W$ couplingsquared. PEW data suggest $m_{C}^{2} \lesssim(200-250 \mathrm{GeV})^{2}$. For multiple Higgs representations of any kind in the most general SUSY context, RGE + perturbativity up to $M_{U} \sim 2 \times 10^{16} \mathrm{GeV}$ implies this same result. Of course, we must remember that many types of new physics at low scale allow evasion of the $m_{C}^{2}$ magnitude constraint; e.g. large extra dimensions or appropriate extra Higgs isospin-breaking structure. We ignore this caveat, assume the sum rule and take $K(m)=$ constant from $m_{A}=m_{h}^{\text {min }}$ to $m_{B}=m_{h}^{\max }: K(m)=1 /\left(m_{B}-m_{A}\right)$. LEP constraints do not allow much weight below $70 \mathrm{GeV}$. For $K(m)=$ constant, $m_{C}=200 \mathrm{GeV}$ and $m_{A}=70 \mathrm{GeV}$ one finds $m_{B}=300 \mathrm{GeV}$ and $m_{B}-m_{A}=230 \mathrm{GeV}$. For the $e^{+} e^{-} \rightarrow Z X$ process at the LC, a fraction $f=100 \mathrm{GeV} / 230 \mathrm{GeV} \sim 0.43$ of the continuum Higgs signal would then lie in the $100 \leq M_{X} \leq 200 \mathrm{GeV}$ mass range (which range avoids the $Z$ resonance region with the largest background and has little phase space suppression at $\sqrt{s}=500 \mathrm{GeV}$ ). Summing 
$Z \rightarrow e^{+} e^{-}+\mu^{+} \mu^{-}$, yields signal and background rates of $S \sim 540 f$ and $B=1080$, for the $100 \leq M_{X} \leq 200 \mathrm{GeV}$ window, assuming $L=200 \mathrm{fb}^{-1}$. The result is

$$
\frac{S}{\sqrt{B}} \sim 16 f\left(\frac{L}{200 \mathrm{fb}^{-1}}\right) \text { for } m \in[100-200] \mathrm{GeV} .
$$

Detecting such a signal will be straightforward. With $L=500 \mathrm{fb}^{-1}$, after a few years we will be able to determine the signal magnitude with reasonable error $(\sim 15 \%)$ in each $10 \mathrm{GeV}$ interval.

In contrast, it appears that hadron collider detection of such a continuum signal is very challenging. In particular, if the Higgs bosons all share the $V V$ coupling, the $\gamma \gamma$ (and $Z Z \rightarrow 4 \ell$ ) excellent resolution modes are likely to fail. Further, the Higgs bosons with some $W W / Z Z$ coupling decay in a manner that cannot be predetermined. If their only decays were to the usual $b \bar{b}, \tau^{+} \tau^{-}, W W^{(*)}, Z Z^{(*)}, \ldots$ channels, Ref. [i] $\left.{ }^{4} \overline{4}\right]$ argues that a signal would be present in the $W W \rightarrow h \rightarrow W W$ channel coming from that part of the continuum with $m \sim 2 m_{W}$. However, this argument does not allow for decays of the CPeven $h$ 's to $V+$ Higgs, to Higgs+Higgs (e.g. $a a$ as in the NMSSM), or to SUSY final states, including the invisible $\widetilde{\chi}_{1}^{0} \widetilde{\chi}_{1}^{0}$ mode. Still, even if some of these latter decays are present one should not abandon all hope of seeing a signal. For example, the $h \rightarrow a a \rightarrow j j \tau^{+} \tau^{-}$ signal is quite strong and tends to accumulate in the same $M_{j j \tau^{+} \tau^{-}}$mass region for a range of $m$ values (roughly, 90 to $150 \mathrm{GeV}$ ). Thus, even if it is reduced by the factor of $60 / 230$ appropriate for the $K(m)=$ constant choice from 70 to $300 \mathrm{GeV}$, it would remain observable. However, this assumes that only a single $a a$ final state is relevant. If there are multiple $a a$ and / or $h^{\prime} h^{\prime}$ final states present in the $h$ decays and the secondary Higgs are not close in mass, the signal strength for any one would be reduced. Most insidious would be cases in which the $h$ decays to $W W, a a$ and $\widetilde{\chi}_{1}^{0} \widetilde{\chi}_{1}^{0}$ all had similar branching ratios implying reduced signals in any one type of mode.

\subsection{Left-right symmetric supersymmetric models}

Proliferation of Higgs bosons is also typical of left-right symmetric supersymmetric (SU-

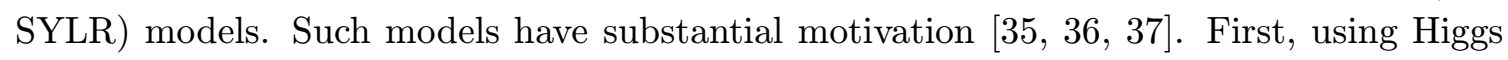
fields to break parity at some high scale $m_{R}$ is an attractive idea. A favorite unified group is $S O(10)$. It automatically includes $\nu_{R}$ fields for neutrino masses as well as the usual $S U(5)$ representation structures, and contains the subgroup $S U(2)_{L} \times S U(2)_{R} \times U(1)_{B-L} \times$ $S U(3)_{C}$. Second, the SUSYLR context guarantees that R-parity is conserved. Third, the SUSYLR structure guarantees that there is no strong CP problem and no SUSY-CP problem (i.e. the generic problem of SUSY phases giving large EDM unless cancellations are carefully arranged) at $m_{R}$. It is then a matter of making sure that evolution from $m_{R}$ down does not destroy these two properties.

The Higgs fields required in one prototype model are listed in Table $13 \overline{3_{r}}$ Two bi-doublets $\Phi$ are required in order to avoid predicting that the CKM matrix is the identity matrix. $S U(2)_{R}$ triplets, $\Delta^{c}$, are employed to break the $S U(2)_{R}$ symmetry. $S U(2)_{L}$ triplets, $\Delta$, are then required by L-R symmetry. 


\begin{tabular}{|c|c|}
\hline Fields & $S U(2)_{L} \times S U(2)_{R} \times U(1)_{B-L}$ representation \\
\hline$Q$ & $(2,1,1 / 3)$ \\
$Q^{c}$ & $(1,2,-1 / 3)$ \\
$L$ & $(2,1,-1)$ \\
$L^{c}$ & $(1,2,+1)$ \\
$\Phi_{1,2}$ & $(2,2,0)$ \\
$\Delta$ & $(3,1,+2)$ \\
$\bar{\Delta}$ & $(3,1,-2)$ \\
$\Delta^{c}$ & $(1,3,+2)$ \\
$\bar{\Delta}^{c}$ & $(1,3,-2)$ \\
\hline
\end{tabular}

Table 3: Fields of sample model

I briefly summarize how it is that such a model avoids the strong and SUSY CP problems. The strong CP phase is defined by the combination

$$
\bar{\Theta}=\Theta+\operatorname{Argdet}\left(M_{u} M_{d}\right)-3 \operatorname{Arg}\left(m_{\widetilde{g}}\right)
$$

where $\Theta$ is the coefficient of the (P-violating) $F_{\mu \nu} \widetilde{F}^{\mu \nu}$ term in the Lagrangian, and $\bar{\Theta}$ must be very small to solve the strong CP problem. $\mathrm{P}$ invariance for scales above $m_{R}$ guarantees that $\Theta=0$ above $m_{R}$. Further, L-R transformations imply that $m_{\widetilde{g}}$ must be real above $m_{R}$. Finally, the Yukawa coupling matrices are required to be hermitian by L-R transformations. Consequently, if the bi-doublet Higgs vevs. are real then quark mass matrices are hermitian (not real - reality of the determinant is all that is required) so that the second term above is zero. Of course, it is necessary to show that the Higgs potential does not spontaneously break CP, a result that follows in general for two pairs of Higgs doublets. The weakest point for the model being described is that one must introduce a single non-renormalizable operator $\frac{\lambda}{M}\left[\operatorname{Tr}\left(\Delta^{c} \tau_{m} \bar{\Delta}^{c}\right)\right]^{2}\left(\Delta^{c}\right.$ 's are the $S U(2)_{R}$ triplet Higgs fields and $M=M_{\mathrm{P}}$ or $\left.m_{R}\right)$ in order that $\left\langle\widetilde{\nu}_{R}\right\rangle=0$ in the vacuum state. Another requirement is imposed on the model in order that significant $\bar{\Theta} \neq 0$ does not develop when evolving below $m_{R}$ (where the $S U(2)_{R}$ gaugino loop no longer cancels the $S U(2)_{L}$ gaugino loop). This is achieved if the theory is constructed so that $S U(2)_{L}$ gaugino masses are real, as can be motivated in $S O(10)$ with suitably generalized L-R symmetry. In the end, the strong CP problem can be solved even if $m_{R}$ is large, as is most natural if the see-saw mechanism is to generate neutrino mass.

Next, consider the SUSY CP problem. Generically speaking, small phases for $A m_{\widetilde{g}}$ and $\mu v_{u} m_{\tilde{g}} / v_{d}$ are required. In SUSYLR models, hermiticity of the $A_{u}$ and $A_{d}$ (soft-SUSYbreaking) terms and of the Yukawa coupling matrices, along with reality of $m_{\tilde{g}}$, guarantee that these phases are small at scales above $m_{R}$. A detailed argument regarding evolution to scales below $m_{R}$ shows that this is maintained to adequate accuracy.

The result is a model with lots of Higgs fields, both triplets and doublets. If $m_{R}$ is large (as motivated by see-saw phenomenology), then only the MSSM two-doublet Higgs sector must necessarily survive at low scales. Still, the only non-MSSM particles of the model are all the Higgs bosons and their SUSY partners, and there is a possibility that some of them could be light. In particular, the $\Delta_{R}$ doubly-charged Higgs and their higgsino partners 
could be the lightest of the non-MSSM particles. It is even not impossible that $m_{R}$ is of order a $\mathrm{TeV}$. In this case, neutrino masses require careful adjustment (small values) of the associated lepton-number violating couplings, but there is very little evolution to possibly mess up the strong CP and SUSY CP solutions and many Higgs will be observable.

\section{Extra Dimensions}

The most natural alternative to employing SUSY to cure the hierarchy problem is to simply lower the Planck scale through the introduction of extra dimensions. There are now many ideas as to how to accomplish EWSB in the extra dimensional context. EWSB can occur via a Higgs sector, via the Scherk-Schwartz mechanism, or via appropriate boundary conditions. Perturbativity for $W_{L} W_{L} \rightarrow W_{L} W_{L}$ scattering can even be achieved using Kaluza-Klein excitations instead of Higgs exchanges to cancel the bad high-energy behavior

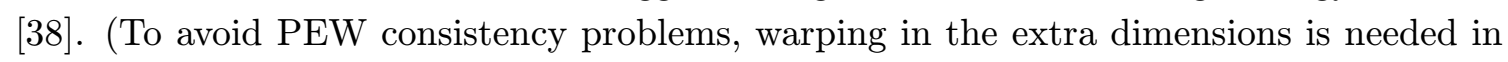
this model.) The simplest models in which EWSB is accomplished via a Higgs sector are those in which all SM particles, including the Higgs boson(s), are confined to a 3-brane (i.e. they are restricted to the usual 3 spatial dimensions and 1 time dimension) and only gravity propagates in the extra dimensions. One possibility is that there are at least two extra flat dimensions. Another simple possibility is to allow for a single extra dimension that is warped. This latter possibility was introduced by Randall and Sundrum [i] $\overline{3} \overline{9}]$ and is the one I consider here. The simplest model is that in which there is one Higgs doublet on the SM brane. The phenomenology of this model has been extensively studied, both without Higgs-radion mixing $\left[\overline{4} \overline{4} \overline{2}, \overline{4} \overline{3}_{1}, \overline{4} \overline{4}, \overline{4}, \overline{4} \overline{5}_{1}^{1}, \overline{4} \overline{6} \overline{6}\right]$ and in the presence of Higgs-radion mixing



\subsection{The Randall-Sundrum, mixed Higgs-radion scenario}

In the RS model, there are two branes, separated in the 5th dimension $(y)$ and $y \rightarrow-y$ symmetry is imposed. With appropriate boundary conditions, the 5D Einstein equations are consistent with the metric

$$
d s^{2}=e^{-2 \sigma(y)} \eta_{\mu \nu} d x^{\mu} d x^{\nu}-b_{0}^{2} d y^{2},
$$

where $\sigma(y) \sim m_{0} b_{0}|y|$. Here, $e^{-2 \sigma(y)}$ is the warp factor. Due to this warping, scales at $y=0$ of order $M_{\mathrm{P}}$ on the hidden brane are reduced to scales at $y=1 / 2$ of order TeV on the visible brane. Fluctuations of $g_{\mu \nu}$ relative to $\eta_{\mu \nu}$ are the KK excitations $h_{\mu \nu}^{n}$. Fluctuations of $b(x)$ relative to $b_{0}$ define the radion field. In addition, we place a Higgs doublet $\widehat{H}$ on the visible brane. After various rescalings, the properly normalized quantum fluctuation field is called $h_{0}$. The radion is stabilized by introducing a radion mass by hand. A possible

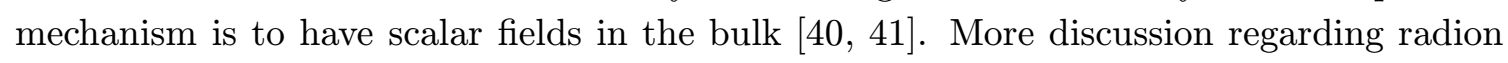
stabilization will appear later.

In general, the Higgs scalar will mix with the radion via a term of the form

$$
S_{\xi}=\xi \int d^{4} x \sqrt{g_{\text {vis }}} R\left(g_{\text {vis }}\right) \widehat{H}^{\dagger} \widehat{H}
$$


where $R\left(g_{\mathrm{vis}}\right)$ is the Ricci scalar for the metric induced on the visible brane. In the end, just four independent parameters completely fix the mass diagonalization of the scalar sector when $\xi \neq 0$. These are:

$$
\xi, \quad \gamma, m_{h}, m_{\phi},
$$

where $\gamma \equiv v_{0} / \Lambda_{\phi}$, with $\Lambda_{\phi}\left(v_{0}=246 \mathrm{GeV}\right)$ being the vacuum expectation value of the bare radion (Higgs) fields. Observation of the 1st KK excitation resonance spectrum at the LHC (as very likely possible) will fix $\Lambda_{\phi}$.

After writing out the full quadratic structure of the Lagrangian, including $\xi \neq 0$ mixing, we obtain a form in which the $h_{0}$ and $\phi_{0}$ fields for $\xi=0$ are mixed $\left(h_{0}=d h+c \phi\right.$, $\left.\phi_{0}=a \phi+b h\right)$ and have complicated kinetic energy normalization. We must diagonalize the kinetic energy and rescale to get canonical normalization. Given $m_{h}$ and $m_{\phi}$ we must invert the mixing equations. The process of inversion is very critical to the phenomenology and somewhat delicate. The result found is that the physical mass eigenstates $h$ and $\phi$ cannot be too close to being degenerate in mass, depending on the precise values of $\xi$ and $\gamma$; extreme degeneracy is allowed only for small $\xi$ and/or $\gamma$.

The phenomenology of the model is determined by the Higgs and radion couplings. Those to $f \bar{f}$ and $V V(V=W, Z)$ are easily related to the SM Higgs couplings:

$$
g_{Z Z h}=\frac{g m_{Z}}{c_{W}}(d+\gamma b), \quad g_{Z Z \phi}=\frac{g m_{Z}}{c_{W}}(c+\gamma a) ;
$$

the $W W$ couplings are obtained by replacing $g m_{Z} / c_{W}$ by $g m_{W}$; and

$$
g_{f \bar{f} h}=-\frac{g m_{f}}{2 m_{W}}(d+\gamma b), \quad g_{f \bar{f} \phi}=-\frac{g m_{f}}{2 m_{W}}(c+\gamma a) .
$$

Note that the same factors relative to SM couplings appear for the $Z Z, W W$ and $f \bar{f}$ couplings: we use the notation $g_{f V h} \equiv d+\gamma b$ and $g_{f V \phi} \equiv c+\gamma a$.

Only the $g g$ and $\gamma \gamma$ couplings are special. First, there are the standard loop contributions, except rescaled by the $f \bar{f} / V V$ strength factors $g_{f V h}$ or $g_{f V \phi}$. In addition, there are anomalous couplings for the bare radion field; these are expressed in terms of the $S U(3) \times S U(2) \times U(1) \beta$ function coefficients $b_{3}=7, b_{2}=19 / 6$ and $b_{Y}=-41 / 6$. The anomalous couplings of the mass eigenstates $h$ and $\phi$ enter only through their radion admixtures.

For purposes of discussing the phenomenology, I will focus on the specific choices $m_{h}=120 \mathrm{GeV}$ and $\Lambda_{\phi}=5 \mathrm{TeV}$. The basic hourglass shape that defines the theoretically allowed region in $\left(\xi, m_{\phi}\right)$ parameter space is illustrated in Fig. 15it, along with contours of $g_{f V h}^{2}$. Observe that $g_{f V h}^{2}<1$ if $m_{\phi}>m_{h}$ and vice versa. Contours of $g_{f V h}^{2}$ are shown in Fig. ' i $\overline{6}_{i}^{i}$ There, we observe that substantial $g_{f V \phi}^{2}$ is possible if $m_{\phi}>m_{h}$ and $\xi$ is not too small. However, $g_{f V \phi}^{2}$ is generically quite small and even exhibits a line of zeroes.

I now outline the capabilities for Higgs and radion detection at the LHC. The precision EW studies suggest that some of the larger $|\xi|$ range is excluded, but we studied the whole

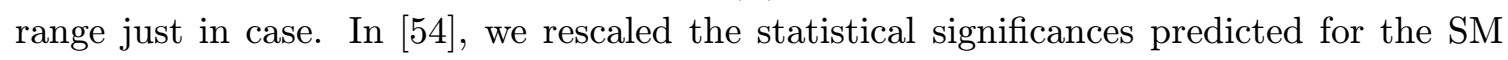
Higgs boson at the LHC using the $h$ and $\phi$ couplings predicted relative to the $h_{\mathrm{SM}}$. The most important modes are $g g \rightarrow h \rightarrow \gamma \gamma$ and $g g \rightarrow \phi \rightarrow Z Z^{(*)} \rightarrow 4 \ell$. Also useful are $t \bar{t} h$ with 


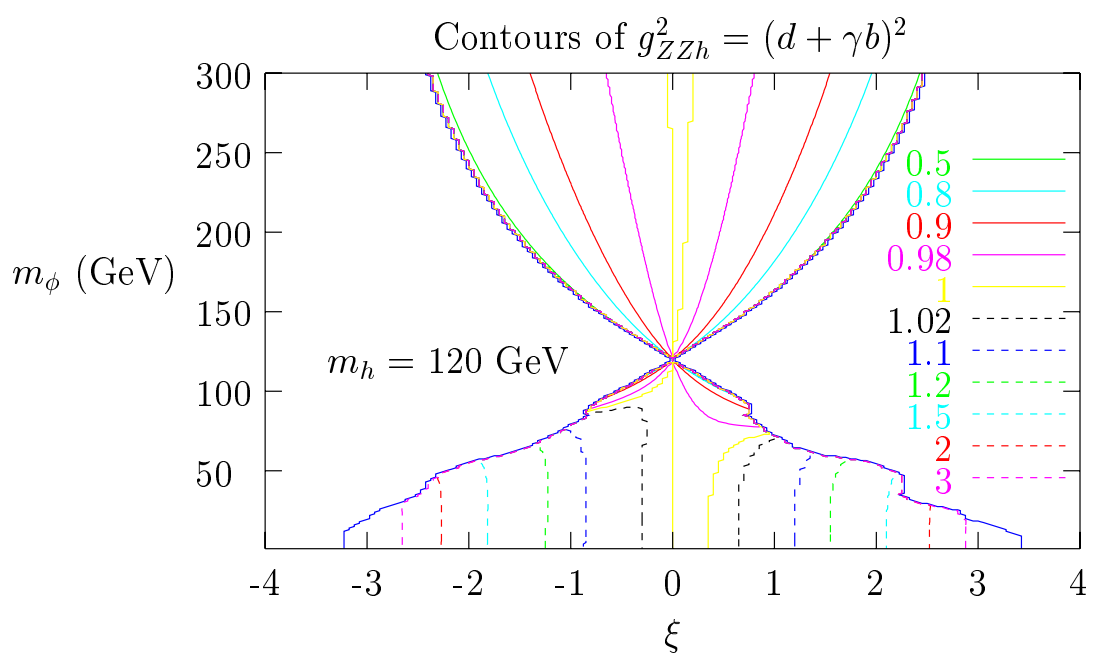

Figure 5: Contours of $g_{f V h}^{2}$ (relative to the SM Higgs boson) for $\Lambda_{\phi}=5 \mathrm{TeV}, m_{h}=120 \mathrm{GeV}$.

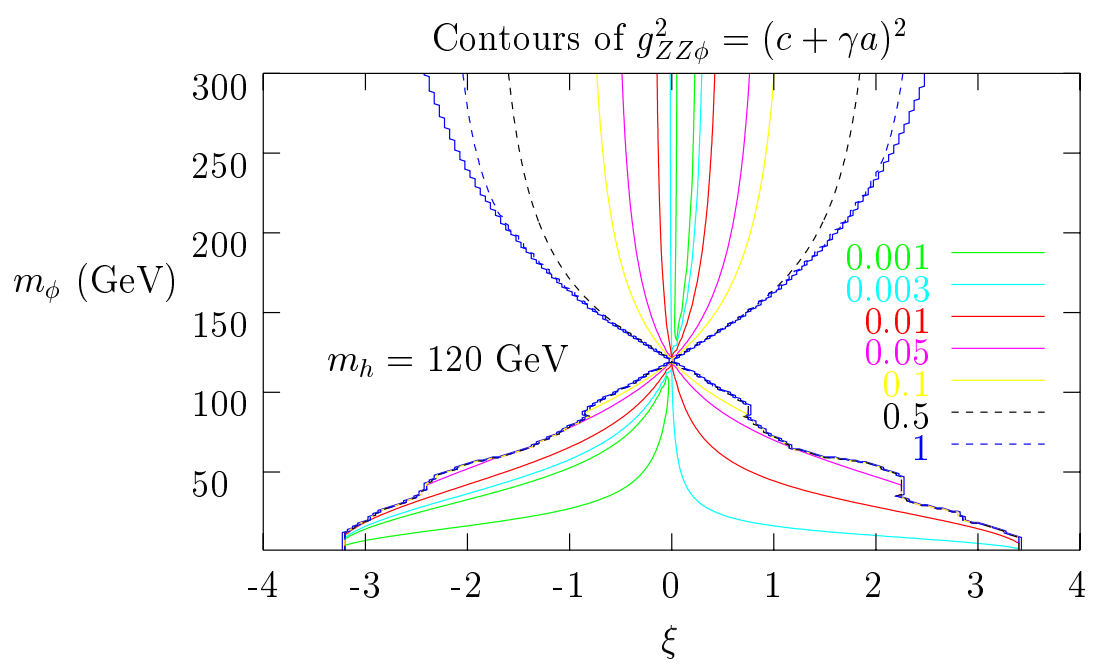

Figure 6: Contours of $g_{f V \phi}^{2}$ for $\Lambda_{\phi}=5 \mathrm{TeV}, m_{h}=120 \mathrm{GeV}$.

$h \rightarrow b \bar{b}$ and $g g \rightarrow h \rightarrow Z Z^{*} \rightarrow 4 \ell$. An example of the type of effect that will be observed is that the $h \rightarrow \gamma \gamma$ mode becomes unobservable if $|\xi|$ is large and $m_{\phi}>m_{h}$ (which together imply suppressed $h W W$ coupling and hence suppressed $W$-loop contribution to the $\gamma \gamma h$ couplings). Fig. $\bar{T}_{1}$ shows that there are regions where the $h$ cannot be detected and that these regions grow (decrease) as $m_{h}$ decreases (increases). The region of unobservability diminishes as $m_{h}$ increases since the $g g \rightarrow h \rightarrow 4 \ell$ increases in strength at higher $m_{h}$.

Figure ${ }_{i=1}^{\bar{s}}$ shows the regions of $h$ observability or unobservability in comparison to regions where $\phi$ can be detected. We see that there are regions of $\left(m_{h}, \xi\right)$ parameter space in which both the $h$ and $\phi$ mass eigenstates will be detectable. In these regions, the LHC will observe two scalar bosons somewhat separated in mass, with the lighter (heavier) having a non-SM-like rate for the $g g$-induced $\gamma \gamma(Z Z)$ final state. Additional information will be required to ascertain whether these two Higgs bosons derive from a multi-doublet or other 

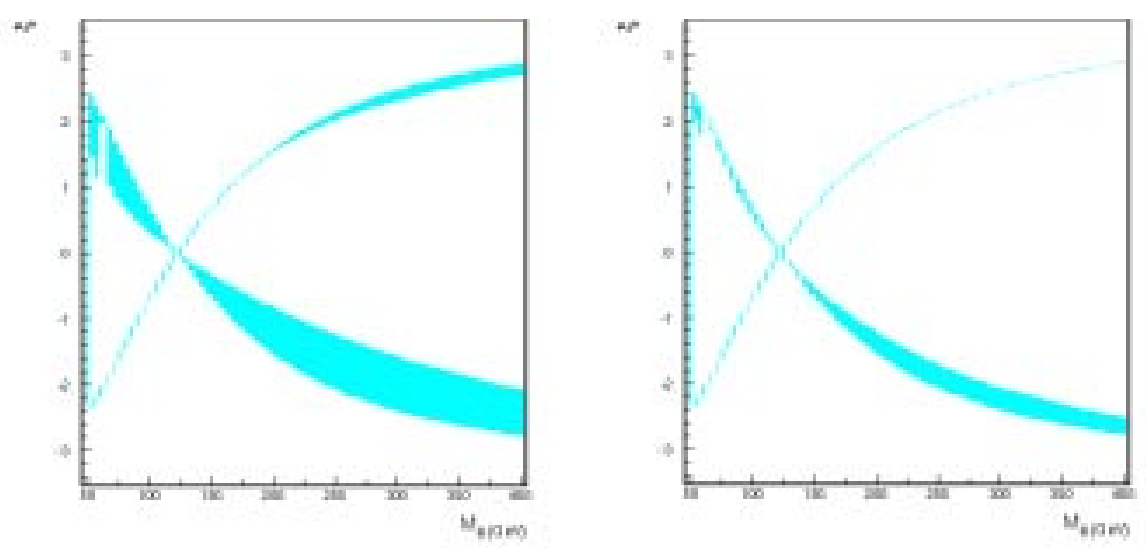

Figure 7: The shaded (cyan) regions are those where $h$ discovery is not possible for the $\Lambda_{\phi}=5 \mathrm{TeV}$ and $m_{h}=120 \mathrm{GeV}$ case assuming LHC luminosity of $L=30 \mathrm{fb}^{-1}$ (left) or $L=100 \mathrm{fb}^{-1}$ (right). Regions outside the outermost hourglass boundary are theoretically excluded.
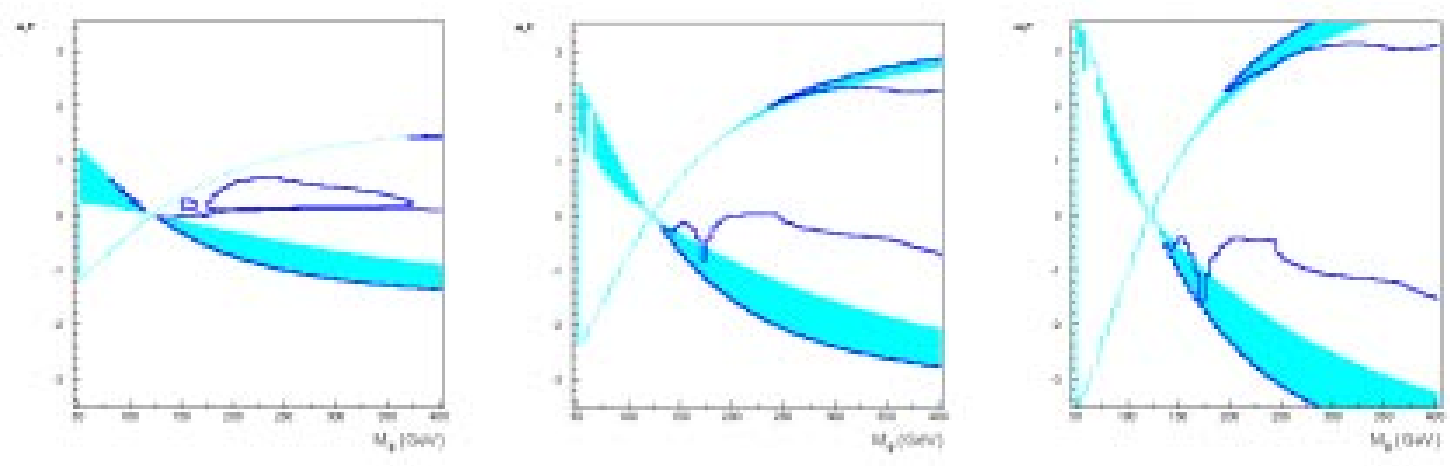

Figure 8: $L=30 \mathrm{fb}^{-1}$ illustration of mode complementarity at the LHC for $m_{h}=120 \mathrm{GeV}$. The shaded (cyan) regions show the regions where neither the $g g \rightarrow h \rightarrow \gamma \gamma$ mode nor the (not very important at this $m_{h}$ value) $g g \rightarrow h \rightarrow 4 \ell$ mode yields a $>5 \sigma$ signal. The regions between dark blue curves define the regions where $g g \rightarrow \phi \rightarrow 4 \ell$ is $>5 \sigma$. The graphs are for $\Lambda_{\phi}=2.5 \mathrm{TeV}$ (left), 5.0 TeV (center), and 7.5 TeV (right).

type of extended Higgs sector or from the present type of model with Higgs-radion mixing.

The LHC results can be compared to possibilities at the linear collider. An $e^{+} e^{-}$ LC would guarantee observation of both the $h$ and the $\phi$ in the region of low $m_{\phi}$, large $\xi>0$ within which detection of either at the LHC might be difficult. This is because, relative to the SM, the $Z Z h$ coupling-squared is always fairly substantial and even the $Z Z \phi$ coupling-squared is $\gtrsim 0.01$ relative to the SM for most of this region, as shown in Fig. $\underline{\underline{p}}_{1}^{\prime}$

Of course, it is now appearing quite likely that the LC will not be built until after the LHC has been running for a number of years. Thus, we must ask if there are alternatives that might allow Higgs and/or radion discovery for parameter regions where the LHC 
signals would be weak or undetectable. One option that might emerge is a $\gamma \gamma$ collider built based on the construction of two modules of the CLIC type. Such test modules are scheduled for relatively earlier completion, and will have sufficient energy to produce a light Higgs boson or light radion.

In [55.], we demonstrated that the $\gamma \gamma$ collider will allow $h$ discovery (for $m_{h}=120 \mathrm{GeV}$ ) throughout the entire hourglass, which is something the LHC cannot do. However, if $m_{\phi}<120 \mathrm{GeV}$ then the $\phi$ is very likely to elude discovery at the $\gamma \gamma$ collider. (Recall that it also eludes discovery at the LHC for this region.) The only exceptions to this latter statement occur at the very largest $|\xi|$ values for $m_{\phi} \geq 55 \mathrm{GeV}$.

There is a big part of the hourglass where a $120 \mathrm{GeV} h$ will be seen in $\gamma \gamma \rightarrow h \rightarrow b \bar{b}$ at the $\gamma \mathrm{C}$ and in $g g \rightarrow h \rightarrow \gamma \gamma$ at the LHC. This is most of the hourglass when $L$ at the LHC is $>100 \mathrm{fb}^{-1}$. The ratio of the rates gives us $\frac{\Gamma(h \rightarrow g g)}{\Gamma(h \rightarrow b \bar{b})}$, in terms of which we may compute

$R_{h g g} \equiv\left[\frac{\Gamma(h \rightarrow g g)}{\Gamma(h \rightarrow b \bar{b})}\right]\left[\frac{\Gamma(h \rightarrow g g)}{\Gamma(h \rightarrow b \bar{b})}\right]_{S M}^{-1}$.

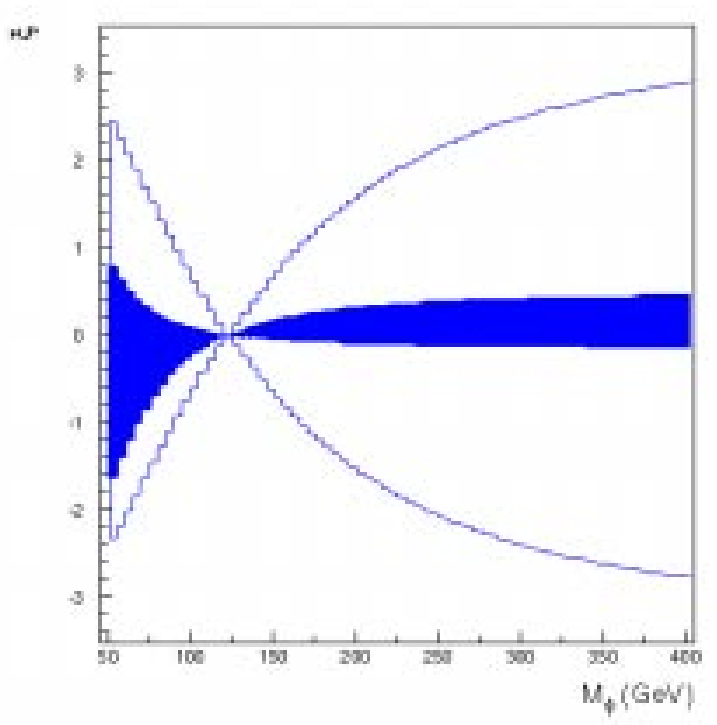

Figure 9: The region in $\left(m_{\phi}, \xi\right)$ parameter space with $g_{\phi Z Z}^{2} / g_{H Z Z}^{2}<0.01$ (implying that the $\phi$ will be difficult to detect at the LC) is indicated by the dark region, for $m_{h}=120 \mathrm{GeV}$ and $\Lambda_{\phi}=5 \mathrm{TeV}$. The $h$ can be detected at the LC for all $\left(m_{\phi}, \xi\right)$ parameter choices.

This is a very interesting number since it directly probes for the presence of the anomalous $g g h$ coupling. In particular, $R_{h g g}=1$ if the only contributions to $\Gamma(h \rightarrow g g)$ come from quark loops and all quark couplings scale in the same way. A plot of $R_{h g g}$ as a function of $\xi$ for $m_{h}=120 \mathrm{GeV}$ and several $m_{\phi}$ choices appears in Fig. II $\underline{1}_{1}^{\prime \prime}$

To assess our ability to determine $R_{g g h}$ from data, we recall that $\Gamma(h \rightarrow \gamma \gamma) \Gamma(h \rightarrow$ $b \bar{b}) / \Gamma_{\text {tot }}^{h}$ can be measured with an accuracy of about $\sim 0.035$. The dominant error will then be from the LHC which will typically measure $\Gamma(h \rightarrow g g) \Gamma(h \rightarrow \gamma \gamma) / \Gamma_{\text {tot }}^{h}$ with an accuracy of between 0.1 and 0.2 (depending on parameter choices and available $L$ ). From Fig. '1 $10_{1}^{\prime}$, we see that 0.2 fractional accuracy will reveal deviations of $R_{h g g}$ from 1 for all but the smallest $\xi$ values. Thus, the ability to measure $R_{h g g}$ may be the strongest reason in the Higgs context for having the $\gamma C$ as well as the LHC. Almost all non-SM Higgs theories predict $R_{h g g} \neq 1$ for one reason another, unless one is in the decoupling limit.

Depending on $L$ at the LHC, there is a somewhat smaller part of the hourglass (large $|\xi|$ with $m_{\phi}>m_{h}$ ) where only the $\phi$ will be seen at the LHC and the $h$ will only be seen at the $\gamma C$. (We don't know for sure about the $\phi$ at the $\gamma C$ until $W W, Z Z$ final states 

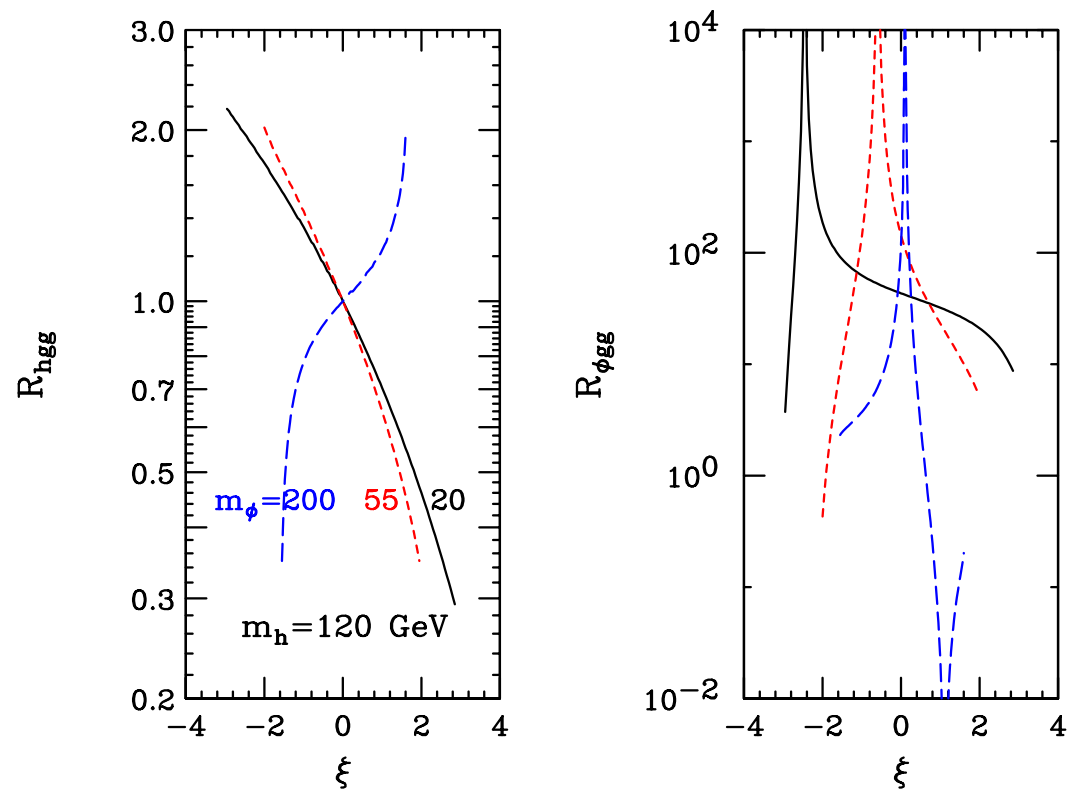

Figure 10: We give the ratios $R_{h g g}$ and $R_{\phi g g}$ of the $h g g$ and $\phi g g$ couplings-squared including the anomalous contribution to the corresponding values expected in its absence. Results are shown for $m_{h}=120 \mathrm{GeV}$ and $\Lambda_{\phi}=5 \mathrm{TeV}$ as functions of $\xi$ for $m_{\phi}=20,55$ and $200 \mathrm{GeV}$. (The same type of line is used for a given $m_{\phi}$ in the right-hand figure as is used in the left-hand figure.)

are studied, but I am not all that optimistic.) This is a nice example of complementarity between the two machines. By having both machines, we maximize the chance of seeing both the $h$ and $\phi$.

Thus, there is a strong case for the $\gamma C$ in the RS model context, especially if a Higgs boson is seen at the LHC that has non-SM-like rates in one or more of the standard detection channels.

\subsection{Complications within the RS model}

Introduction of a mass for the radion either by hand or via the Goldberger-Wise approach leads to perturbations of the exact RS metric and/or curvature of the branes. However, if one introduces a bulk scalar with carefully tuned brane and bulk potential, it is possible to obtain a mass for the radion while retaining the RS metric as an exact solution [5 $\left.\bar{\sigma}_{-1}\right]$. The KK excitations of the bulk scalar then mix with the radion and the Higgs and the phenomenology could potentially become more difficult. In some limits, one can imagine getting a "warped" version of the multi-Higgs type of scenarios.

\section{An alternative discovery technique: $p p \rightarrow p p h($ or $\phi)$}

It is claimed that doubly-elastic scalar production at the LHC will give a high-resolution

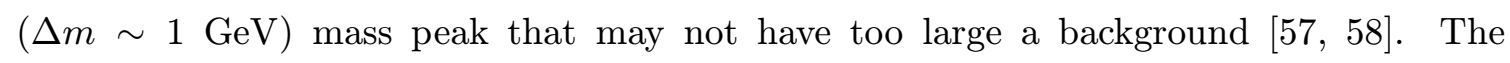
production rates are substantial for lighter Higgs bosons in the CPX scenario. Rates for the more general Higgs bosons we have considered and for the radion have not been 
computed yet. If a doubly-elastic signal can be established, it would obviously play an important role in sorting out the type of models we have been discussing.

\section{Conclusions}

It seems almost certain the the SM and its simple one-doublet Higgs sector will not describe $\mathrm{TeV}$ scale physics. There are strong motivations for extending the model in a variety of exciting and unusual ways, all of which have dramatic implications. In those extensions that implement EWSB in the context of a Higgs sector of some sort, the phenomenology associated with the discovery of the Higgs bosons and possibly mixed radion(s) is both complex and challenging. Here, I have reviewed just a few of the attractive possibilities that have been discussed in the literature. They illustrate the need for flexibility in the analysis procedures and an extensive data set (not only from the LHC, but also from a future LC and $\gamma \mathrm{C}$ ) in order to be certain of discovering even one Higgs boson and certainly in order to fully unravel the complete Higgs sector.

\section{References}

[1] J. F. Gunion, R. Vega and J. Wudka, Phys. Rev. D 43, 2322 (1991).

[2] R. N. Mohapatra and G. Senjanovic, Phys. Rev. D 23, 165 (1981).

[3] J. F. Gunion, Int. J. Mod. Phys. A 13, 2277 (1998) [arXiv:hep-ph/9803222].

[4] N. G. Deshpande, J. F. Gunion, B. Kayser and F. I. Olness, Phys. Rev. D 44, 837 (1991).

[5] P. Chankowski, T. Farris, B. Grzadkowski, J. F. Gunion, J. Kalinowski and M. Krawczyk, Phys. Lett. B 496, 195 (2000) [arXiv:hep-ph/0009271].

[6] The results of Fig. 1ij were provided by E. Richter-Was and F. Gianotti on behalf of the ATLAS collaboration.

[7] D. Denegri et al., "Summary of the CMS Discovery Potential for the MSSM SUSY Higgses," CMS NOTE 2001/032 [arXiv:hep-ph/0112045].

[8] B. Grzadkowski, J. F. Gunion and J. Kalinowski, Phys. Lett. B 480, 287 (2000) [arXiv:hep-ph/0001093].

[9] A. Djouadi, J. Kalinowski and P. M. Zerwas, Mod. Phys. Lett. A 7, 1765 (1992).

[10] T. Farris, J. F. Gunion and H. E. Logan, in Proc. of the APS/DPF/DPB Summer Study on the Future of Particle Physics (Snowmass 2001) ed. N. Graf, eConf C010630, P121 (2001) [arXiv:hep-ph/0202087].

[11] J. F. Gunion, in Proc. of the APS/DPF/DPB Summer Study on the Future of Particle Physics (Snowmass 2001) ed. N. Graf, eConf C010630, P116 (2001) [arXiv:hep-ph/0110362].

[12] D. M. Asner, J. B. Gronberg and J. F. Gunion, Phys. Rev. D 67, 035009 (2003) [arXiv:hep-ph/0110320].

[13] J. R. Forshaw, arXiv:hep-ph/0305162.

[14] C. F. Kolda and H. Murayama, JHEP 0007, 035 (2000) [arXiv:hep-ph/0003170]. 
[15] N. Arkani-Hamed, A. G. Cohen and H. Georgi, Phys. Lett. B 513, 232 (2001) [arXiv:hep-ph/0105239].

[16] H. E. Logan, arXiv:hep-ph/0310151.

[17] J.F. Gunion, H.E. Haber, G. Kane and S. Dawson, The Higgs Hunter's Guide (Perseus Publishing, Reading, MA, 2000).

[18] See the LEP Higgs Working Group web pages, http://lephiggs . web.cern.ch/LEPHIGGS, especially LHWG Note/2001-04 (2001).

[19] M. Carena, J. M. Moreno, M. Quiros, M. Seco and C. E. M. Wagner, Nucl. Phys. B 599, 158 (2001) [arXiv:hep-ph/0011055].

[20] M. Carena, M. Quiros, M. Seco and C. E. M. Wagner, Nucl. Phys. B 650, 24 (2003) [arXiv:hep-ph/0208043].

[21] H. Murayama and A. Pierce, Phys. Rev. D 67, 071702 (2003) [arXiv:hep-ph/0201261].

[22] S. Davidson, T. Falk and M. Losada, Phys. Lett. B 463, 214 (1999) [arXiv:hep-ph/9907365].

[23] J. R. Ellis, K. A. Olive, Y. Santoso and V. C. Spanos, arXiv:hep-ph/0310356.

[24] M. Carena, J. R. Ellis, A. Pilaftsis and C. E. M. Wagner, Nucl. Phys. B 586, 92 (2000) [arXiv:hep-ph/0003180].

[25] J. R. Ellis, J. F. Gunion, H. E. Haber, L. Roszkowski and F. Zwirner, Phys. Rev. D 39, 844 (1989).

[26] M. Cvetic, L. L. Everett, P. Langacker and J. Wang, arXiv:hep-ph/9902247.

[27] A. E. Nelson, N. Rius, V. Sanz and M. Unsal, arXiv:hep-ph/0211102.

[28] J. F. Gunion, H. E. Haber and T. Moroi, arXiv:hep-ph/9610337.

[29] B. A. Dobrescu, G. Landsberg and K. T. Matchev, Phys. Rev. D 63, 075003 (2001) [arXiv:hep-ph/0005308].

[30] U. Ellwanger, J. F. Gunion and C. Hugonie, arXiv:hep-ph/0111179.

[31] U. Ellwanger, J. F. Gunion, C. Hugonie and S. Moretti, arXiv:hep-ph/0305109, and work in progress.

[32] M. Carena, J. R. Ellis, S. Mrenna, A. Pilaftsis and C. E. M. Wagner, Nucl. Phys. B 659, 145 (2003) [arXiv:hep-ph/0211467].

[33] J. R. Espinosa and J. F. Gunion, Phys. Rev. Lett. 82, 1084 (1999) [arXiv:hep-ph/9807275].

[34] A. Alves, O. Eboli, T. Plehn and D. Rainwater, arXiv:hep-ph/0309042.

[35] R. N. Mohapatra and A. Rasin, Phys. Rev. D 54, 5835 (1996) [arXiv:hep-ph/9604445].

[36] R. N. Mohapatra, A. Rasin and G. Senjanovic, Phys. Rev. Lett. 79, 4744 (1997) [arXiv:hep-ph/9707281].

[37] K. S. Babu, B. Dutta and R. N. Mohapatra, Phys. Rev. D 65, 016005 (2002) [arXiv:hep-ph/0107100].

[38] C. Csaki, C. Grojean, L. Pilo and J. Terning, arXiv:hep-ph/0308038.

[39] L. Randall and R. Sundrum, Phys. Rev. Lett. 83, 3370 (1999) [arXiv:hep-ph/9905221]. 
[40] W. D. Goldberger and M. B. Wise, Phys. Rev. Lett. 83, 4922 (1999) [arXiv:hep-ph/9907447].

[41] W. D. Goldberger and M. B. Wise, Phys. Lett. B 475, 275 (2000) [arXiv:hep-ph/9911457].

[42] S. B. Bae, P. Ko, H. S. Lee and J. Lee, Phys. Lett. B 487, 299 (2000) [arXiv:hep-ph/0002224].

[43] H. Davoudiasl, J. L. Hewett and T. G. Rizzo, Phys. Rev. Lett. 84, 2080 (2000) [arXiv:hep-ph/9909255].

[44] K. Cheung, Phys. Rev. D 63, 056007 (2001) [arXiv:hep-ph/0009232].

[45] H. Davoudiasl, J. L. Hewett and T. G. Rizzo, Phys. Rev. D 63, 075004 (2001) [arXiv:hep-ph/0006041].

[46] S. C. Park, H. S. Song and J. Song, Phys. Rev. D 63, 077701 (2001) [arXiv:hep-ph/0009245].

[47] G. Giudice, R. Rattazzi, J. Wells, Nucl. Phys. B595 (2001), 250, hep-ph/0002178.

[48] C. Csaki, M.L. Graesser, G.D. Kribs, Phys. Rev. D63 (2001), 065002-1, hep-th/0008151.

[49] T. Han, G. D. Kribs and B. McElrath, Phys. Rev. D 64, 076003 (2001) [arXiv:hep-ph/0104074].

[50] M. Chaichian, A. Datta, K. Huitu and Z. h. Yu, Phys. Lett. B 524, 161 (2002) [arXiv:hep-ph/0110035]. These authors (see their Appendix A) employ the same full mixing procedure as that employed in Refs. [5

[51] J. L. Hewett and T. G. Rizzo, JHEP 0308, 028 (2003) [arXiv:hep-ph/0202155]. As pointed out in their revision, dated July 2, 2003, Ref. [533] appeared 4 months after their original hep-ph submission. However, prior to the revision their results were incorrect except at rather small $\xi$ values since they employed the small $\xi$ approximations to the mixing / diagonalization procedures given in [4 $\left.\bar{u}^{2}\right]$. The revision of July 2, 2003 comes some 12 months after the Ref. [5

[52] C. Csaki, M. Graesser, L. Randall and J. Terning, Phys. Rev. D 62, 045015 (2000) [arXiv:hep-ph/9911406].

[53] D. Dominici, B. Grzadkowski, J. F. Gunion and M. Toharia, Nucl. Phys. B 671, 243 (2003) [arXiv:hep-ph/0206192].

[54] M. Battaglia, S. De Curtis, A. De Roeck, D. Dominici and J. F. Gunion, Phys. Lett. B 568, 92 (2003) [arXiv:hep-ph/0304245].

[55] D. Asner et al., arXiv:hep-ph/0308103.

[56] B. Grzadkowski and J. F. Gunion, Phys. Rev. D 68, 055002 (2003) [arXiv:hep-ph/0304241].

[57] A. B. Kaidalov, V. A. Khoze, A. D. Martin and M. G. Ryskin, arXiv:hep-ph/0311023.

[58] B. E. Cox, J. R. Forshaw, J. S. Lee, J. Monk and A. Pilaftsis, Phys. Rev. D 68, 075004 (2003) [arXiv:hep-ph/0303206]. 